\title{
MedBot: Conversational Artificial Intelligence Powered ChatBot for Health Care
}

\author{
Karun Babu $^{1}$, Shahana Nishad ${ }^{2}$, Rahana Nishad ${ }^{3}$, Rose Maria Babu ${ }^{4}$, Tony Thankachan ${ }^{5}$, Libin M Joseph ${ }^{6}$ \\ ${ }^{1}$ PG Student, Department of Computer Applications -Saintgits College of Engineering (Autonomous) Pathamuttom \\ Kottayam Kerala, India, karunbabu.ettupara@gmail.com \\ ${ }^{2}$ PG Student, Department of Computer Applications -Saintgits College of Engineering (Autonomous) Pathamuttom \\ Kottayam Kerala, India,shahananishad064@gmail.com \\ ${ }^{3}$ PG Student, Department of Computer Applications -Saintgits College of Engineering (Autonomous) Pathamuttom \\ Kottayam Kerala, India, rahananishad7560@ gmail.com \\ ${ }^{4}$ PG Student, Department of Computer Applications -Saintgits College of Engineering (Autonomous) Pathamuttom \\ Kottayam Kerala, India,rosemariababu1998@gmail.com \\ ${ }^{5}$ PG Student, Department of Computer Applications -Saintgits College of Engineering (Autonomous) Pathamuttom \\ Kottayam Kerala, India,toneythankachan@gmail.com \\ ${ }^{6}$ Assistant Professor, Department of Computer Applications -Saintgits College of Engineering (Autonomous) Pathamuttom,
}

\begin{abstract}
Healthcare is essential for leading a happy life. However, It is exceedingly difficult to seek a doctor's appointment in the event of a health condition. The suggested solution is to create a medical chatbot that can diagnose the disease and provide basic information about it before consulting a doctor. The medical chatbot was created with the aim of lowering healthcare costs and increasing access to medical information. The medical chatbot was developed with the aim of lowering healthcare costs. This system will improve processes services and outcomes and reduce cash. In a self-service mode, users communicate with the ChatBot through text. Healthcare ChatBot was developed with the aim of maintaining internal documents. It operates as a medical reference guide, assisting patients in learning about their condition and determining the disease's status based on symptoms. The main aspect of the Healthcare ChatBot is that the user can connect with the Bot directly without any restrictions, and that the user can use the app at any time without hesitation and without having to pay any money to learn about their condition. We will add more modules in the future, such as doctors and local hospitals, so that users have a better of what they want to do next. Patients can talk to a text-to-text diagnosis bot about their health conditions, and the bot will give them a customized diagnosis based on their understanding symptoms. As a result, people will be more aware of their health, and they will be better protected.
\end{abstract}

Key words: Artificial Intelligence, ChatBot, Flask, Natural Language Processing

\section{INTRODUCTION}

Typically, users are unaware of all of the treatment options symptoms regarding a particular disease. For small problems Users must visit a hospital for a check-up, which takes more time. Handling phone calls for complaints is also time-consuming. Such a stumbling block is frequently alleviated by using a medical ChatBot that provides proper guidance on healthy living. The user can use the ChatBot to ask any personal question about health care by providing their symptom as input, even if they are not physically present at the hospital. A question is sent to ChatBot, who responds with relevantinformation.HealthcareChatBotprovidepersonalizeda ccesstohealthrelatedinformation'sandinteractionsthroughanat uralconversation experience. This system will improve processes services and outcomes and reduce cash. Users interact with the ChatBot via text in a self-service manner. Healthcare ChatBot is created with a responsibility of internal record keeping. It acts as a medical reference book, which help the patient to know about their disease and helps to find the status of the disease based on the symptoms. The ChatBot can use the dataset to generate responses that are relevant and grammatically correct. The main applicability of Healthcare ChatBot is that the user can directly interact with the Bot without any obstacles, and the user can use the system at anytime without any delay and don't have to spend any amount to know their disease. In future we can add more modules like doctors, nearby hospitals etc, so that the users will get a full idea about what they want to do next. Also, there is a possibility that along with text messages we can add voice messages to interact with the bot. It will reduce the effort taken by the user for the effective communication with bot.

\subsection{Organization Profile SAINTGITS COLLEGE OF ENGINEERING}

Saintgits College of Engineering is a self-supporting technical college in Kerala's Kottayam district. The college was established in the year 2002. Saintgits is affiliated to APJ Abdul Kalam Technological University in Kerala and is approved by an All India Council for Technical Education. The institute is accredited by NBA in 2016 for 3 years for 5 
UG programs and in 2017 for 3 years for Master of Computer Application program.

The college inculcates the development of all facets of the mind culminating in an intellectual and balanced personality. A team of dedicated and caring faculty strives to widen the student's horizon of learning there by achieving excellent results for every student. Saintgits College of Engineering, right from inception, has been maintaining high levels of standards in academic and extra-curricular realms of activities. The college has been the centre of technical education in Kerala, conducting national and state-level seminars and symposiums.

\subsection{Objectives of the project}

Healthcare ChatBot provide personalized access to health related information's and interactions through a natural conversation experience. This system will improve processes services and outcomes and reduce cash. Users interact with the ChatBot via text in an exceedingly self-service manner. The ChatBot service implements Natural Language Processing (NLP) and Artificial Intelligence (AI) technologies to understand the user's intent and supply accurate information. Our project help users with symptoms checking that's the symptom checker help Patients find appropriate care. It enables meaningful conversations for patients with an interactive symptom checker.

\subsection{Purpose, Scope and Applicability of the project}

Healthcare ChatBot is created with a responsibility of internal record keeping. It help the patient to know about their disease and helps to find the status of the disease based on the symptoms. We designed a ChatBot that utilizes a common sense database to provide fast, relevant responses while chatting. With some understanding of the concept, we were able to properly parse human feedback and find the most relevant keywords. The database can be used by the ChatBot to produce appropriate and grammatically correct responses. For this to work, the parsing of the human input must be outstanding. The ChatBot must be able to determine which keywords are important. It will also be difficult to produce responses that appear natural using the database.

In our project, we are focused mainly on a datasets with a number of symptoms for a number of diseases. We can extend this dataset to a higher dataset by increasing the number of symptoms and diseases. So that the checking of symptoms performed in the dataset is also increased. If the dataset becomes very large then any user with any symptom can easily identify their disease without any delay with a high accuracy. And also,there is a provision for adding, deleting and modifying the symptom and diseases. The main applicability of Healthcare ChatBot is that the user can directly interact with the Bot without any obstacles, and the user can use the system at any time without any delay and don't have to spend any amount to know their disease. In future we can add more modules like doctors, nearby hospitals etc, so that the users will get a full idea about what they want to do next. Also, there is a possibility that along with text messages we can add voice messages to interact with the bot. It will reduce the effort taken by the user for the effective communication.

\section{LITERATURE SURVEY}

2.1 A Self-Diagnosis Medical Chatbot using Artificial Intelligence, Author: Divya, Indhumathi, Ishwarya, Priyasankari, Kalpana Devi UG Students, Department of Computer Science and Engineering, Easwari Engineering College, Chennai.

Artificial Intelligence is based on how a machine perceives its environment and takes actions based on the data it perceives in order to achieve a good outcome. It's the research into intelligent agents. When a computer mimics "cognitive" tasks that humans associate with other human brains, such as "learning" and "problem solving," the word "artificial intelligence" is used. Artificial Intelligence gives the supreme power to mimic the human way of thinking and behaving to a computer. A text-to-text diagnosis bot engages patients in conversation about their medical issues and provides a personalized diagnosis based on their symptoms. Hence, people will have an idea about their health and have the right protection.

2.2A Medical ChatBot, Author: Mrs. RashmiDharwadkar1, Dr. Mrs. NeetaA.Deshpande2 PG Student, Department of Computer Engineering, D.Y.Patil College, of Engineering, Akurdi, Pune,India.

The system uses NLP to allow computers to connect with humans, i.e. the recognition of key linguistic relations is completed in order to parse the subject into the object of the sentence. The model is trained using an AIML (Artificial Intelligent Markup Language) framework, and the user's words are defined using a Microsoft voice synthesizer. SVM, a strong classifier that can differentiate between two classes, can be used. It assigns the test image to the class with the greatest distance up to the training's neighboring point. It's a learning algorithm for finding the best distinguishing hyperplane that minimizes the error for patterns that haven't been seen before. Porter Stemming Algorithm (Porter Stemmer) is a procedure for discarding the ordinary words which is related to the exact form of words with also having proper endings in English.

Similarity Measure, $\mathrm{Sr}=1-\||\mathrm{r} 1-\mathrm{r} 2| / / \mid \mathrm{r} 1+\mathrm{r} 2$

\section{OBJECTIVES AND PROPOSED INNOVATION}

\subsection{Broad Objectives}

Healthcare ChatBot provide personalized access to health related information's and interactions through a natural conversation experience. This system will improve processes services and outcomes and reduce cash. Users interact with the ChatBot via text in an exceedingly self- service manner. 
The ChatBot service implements Natural Language Processing (NLP) and Artificial Intelligence (AI) technologies to understand the user's intent and supply accurate information. Our project help users with symptoms checking that's the symptom checker help Patients find appropriate care. It enables meaningful conversations for patients with an interactive symptom checker.

\subsection{Proposed System}

Some ChatBots are miniature medical reference books that are useful not only for doctors and patients, but also for anyone interested in learning more about health. The patient has the impression that they are a part of their own health care. The patient has the impression that they are a part of their own health care. Patients who feel included, and who connect with the healthcare system through ChatBots, are more likely to stick with the system, which is advantageous to them and the healthcare provider.Bots can help with basic health-related questions and disease prediction without the need for human intervention. Customer satisfaction is the most significant element in the success of this method.The actual welfare of the ChatBot is to facilitate the people by giving proper guidance regarding the good and healthy living.

Some of the benefits are:

- Less Time Consuming

- Immediate Response for Patients

- Improve Patient Satisfaction

- Reduce need of documentation

- $\quad$ Easy to use

\subsection{Functional Specifications and User Characteristics}

- The users can directly interact with the ChatBot via their own authorized account.

- If users don't have an account, they can register then they can login to the system.

- Users will get a medical result based on their symptom.

- The answers given to the questions asked by the bot is in the Form of Yes/No.

- $\quad$ Provide a faster and secure way of communication and prediction.

- Improved User Interface-A friendly user interface vastly reduces the various overheads

- Faster loading time and High Performance-to load a page anymore. Increased loading time directly contributes to high performance.

- Extremely helpful user support-User support is provided to the maximum. The user can interact with the bot and the proper disease will be predicted.

\subsection{System Specifications}

\section{SYSTEM SPECIFICATIONS}

- OperatingSystem:Windows10

- $\quad$ Front End:Python

- BackEnd:MySQL Server

- Technology:Flask

- Web Technologies:HTML,CSS

- Database:MySQL
- IDLE: Pycharm Community

HARDWARE SPECIFICATIONS

- Input Device: Mouse, Keyboard

- Output device:Monitor

- Memory:8GBRAM

\section{CONCLUSION}

Scheduling appointments and answering typical queries is what most health care directors pay their day doing. Continuance constant words and actions over and yet again is neither productive nor extremely necessary. This kind of job are often simply performed by a ChatBot, who will collect user requests as symptoms, and answer to it queries. It will permit the users to interact with the system with none time delay.

\section{FUTURE SCOPE OF THE PROJECT}

The tasks for a ChatBot in healthcare are basic now, the potential for them to be used as diagnostic tools and more is obvious. Even at this stage, they're helping reduce staff load and overhead costs, improve patient services and supply a 24/7conversation outlet. In future, through ChatBot interface the users are able to directly interact with doctors by seeing one another employing a video call. This can make the users more convenient and trust to use that system.

\section{REFERENCES}

1. AMedicalChatBot,Author:Mrs.RashmiDharwadkar1,Dr .Mrs.Neeta A. Deshpande2 PG Student, Department of Computer Engineering, D.Y. Patil College, of Engineering, Akurdi, Pune, India.

2. A Self-Diagnosis Medical ChatBot Using Artificial Intelligence, Author: Divya, Indumathi, Ishwarya, Priyasankari, KalpanaDevi UG students.

3. Benilda Eleonor V. Comendador, Bien Michael B. Francisco, Jefferson S.Medenilla, Sharleen Mae T. Nacion, and Timothy Bryle E. Serac, "Pharmabot:A Pediatric Generic Medicine Consultant Chatbot "Journal of Automation and Control Engineering".

4. ChatBots meet eHealth: automatizing healthcare, Author: Flora Amato,Stefano Marrone, Vincenzo Moscato, Gabriele Piantadosi, Antonio Picariello,and CarloSansone.

5. ChatBot using a Knowledge in Database,"BayuSetiaji,FerryWahyuWibowo",2016 7th International Conference on Intelligent Systems, Modelling and Simulation.2016 IEEE.

6. Designing for Health ChatBots, Author:Ahmed Fadhil,Gianluca Schiavo,University of Trento, Emanuela Haller,Traian Rebedea Faculty of Automatic Control and Computers university Politehnica of Bucharest 978-0-7695-4980-4/13 \$26.00 @2013IEEE."Designing a Chat-bot that Simulates a Historical Figure". 